Research Article

\title{
Coronary Angiography Characteristics of Symptomatic Patients with Prior Coronary Artery Bypass Graft: A Descriptive Study
}

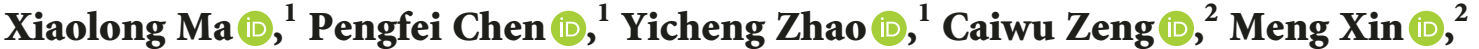 \\ Qing Ye $\mathbb{D}^{3}{ }^{3}$ and Jiangang Wang $\mathbb{D}^{1}$ \\ ${ }^{1}$ Department of Cardiac Surgery, Beijing Anzhen Hospital, Capital Medical University, Beijing, China \\ ${ }^{2}$ Center for Cardiac Intensive Care, Beijing Anzhen Hospital, Capital Medical University, Beijing, China \\ ${ }^{3}$ Department of Cardiac Surgery, Beijing Huaxin Hospital, First Hospital of Tsinghua University, Beijing, China
}

Correspondence should be addressed to Jiangang Wang; jiangangwang@ccmu.edu.cn

Received 28 April 2019; Revised 11 September 2019; Accepted 21 September 2019; Published 11 November 2019

Academic Editor: Vida Demarin

Copyright (C) 2019 Xiaolong Ma et al. This is an open access article distributed under the Creative Commons Attribution License, which permits unrestricted use, distribution, and reproduction in any medium, provided the original work is properly cited.

Objectives. The target of this study was to explore the coronary angiography characteristics for symptomatic patients with prior coronary artery bypass graft (CABG). Methods. Between 2009 and 2017, 993 patients who had undergone CABG but subsequently suffered recurrent symptoms in Beijing Anzhen Hospital were selected for this study and divided into either medical therapy (MT) group $(n=351)$ or percutaneous coronary intervention (PCI) group $(n=642)$ based on the treatment. Clinical data were analyzed between two groups. Results. Patients in the MT group were older and more likely to have chronic lung disease $(6.6 \%$ vs $3.4 \%$, $P=0.026)$ while patients in the PCI group were more likely to have prior MI $(8.8 \%$ vs $17.0 \%, P<0.001)$. In the MTgroup, $54.4 \%$ of patients had newly developed lesions both in the graft and native coronary artery while $58.1 \%$ in the PCI group $(P=0.003)$, and in the MT group, $80.6 \%$ had type C coronary artery disease while $60.1 \%$ in the PCI group $(P<0.001)$. Patients in the MT group presented higher proportion of diffuse lesions $(49.3 \%$ vs $15.0 \%, P<0.001)$ in native coronary arteries. Conclusion. Patients receiving MT (35.3\%) likely had occluded grafts and type C coronary artery disease featuring as diffuse lesions.

\section{Introduction}

For patients with prior coronary artery bypass graft (CABG), the likelihood of experiencing recurrent symptoms has increased over years due to longer life expectancy [1] and the possibility of disease progression of native coronary artery (NCA) or surgical graft failure [2].

Frequent recurrent symptoms in patients with prior CABG usually indicate the occurrence of myocardial ischemia. Treatments for symptomatic patients with prior CABG include revascularization by percutaneous coronary intervention (PCI) or redo CABG and medical therapy (MT). However, repeat revascularization procedures are markedly different from $d e$ novo interventions performed on patients without prior CABG [3]. As a consequence of the specificity of vessel lesions and clinical characteristics in patients with prior CABG [4], previous surgery may increase procedural risk, technical complexity [5], and mortality [6, 7], especially for redo CABG. MT consists of antiplatelet agent, statins agent, $\beta$-blocker, and angiotensin-converting enzyme inhibitor (ACEI) or angiotensin receptor blocker (ARB), considered as optimal medical therapy (OMT) [8]. In fact, clinically, these patients often receive repeat revascularization by PCI for clinical and anatomic factors [2, 9], and patients who could not undergo PCI revascularization would receive MT. However, the coronary angiography (CAG) basis of therapy selection (MT vs PCI) for these patients has been studied in limited data.

In this study, we retrospectively analyzed the clinical data and features of native coronary artery (NCA) lesions and graft lesions by CAG in symptomatic patients with prior CABG who received MT or PCI, aiming to find out CAG characteristics of these patients. 


\section{Methods}

2.1. Study Design. This study was a retrospective observational study conducted in Beijing Anzhen Hospital, Capital Medical University, Beijing Institute of Heart Lung and Blood Vessel Diseases, Beijing, China. We consecutively included symptomatic patients with prior CABG, from 2008 to 2018 in the Department of Cardiology, Anzhen Hospital. Patients with complete data were segregated into the MT or PCI group, depending on the treatment they received. All data were reviewed by one cardiac surgeon and one cardiologist. This study received approval from the Ethics Committee of Beijing Anzhen Hospital.

2.2. Definitions Used in This Study. A graft with the ratio of stenosis diameter to reference vessel diameter $\geq 70 \%$ was defined as stenosis. A graft with stenosis or occlusion was classified as a diseased graft. The classification of ischemic territory was based on CAG results after rehospitalization and also referred to the CAG results prior to CABG. The newly developed lesions were divided into isolated graft disease, NCA and graft disease, and NCA disease.

2.3. Statistical Analysis. All results were analyzed by using the statistical package SPSS 20.0. Categorical variables are presented by raw numbers (\%), and numerical values are presented as means \pm standard deviation. Comparison of the medication and PCI groups was achieved by using Fisher's exact test for each variable and the Mann-WhitneyWilcoxon nonparametric test for continuous variables. For all analyses reported, $P$ values were 2 -sided. Statistical differences were considered significant at $P<0.05$.

\section{Results}

3.1. Patient Population. During the study period, 1456 patients with prior CABG were rehospitalized. Based on the exclusion criteria, 993 patients were included in this study (Figure 1). In each group, symptomatic patients with prior CABG were mainly distributed within 1 to 5 years $(44.4 \%$ vs $46.4 \%$ ) followed by 5 to 10 years (33.3\% vs $31.6 \%)$ after CABG surgery (Table 1). The proportion of patients accepting MT increased as a function of time elapsed after CABG while the proportion of patients accepting PCI treatment decreased (Figure 2).

3.2. Baseline Characteristics. Baseline characteristics are shown in Table 2. Compared with patients in the PCI group, patients in the MT group were older and more likely to have chronic lung disease while patients in the PCI group were more likely to have prior MI. There were no significant differences in comorbidities like diabetes, hypertension, dyslipidemia, prior PVD, prior CVA, prior HF, or prior PCI between two groups. In each group, most patients had recurrent symptoms featuring as chest pain. In both groups, some patients failed to persist in medical therapy like aspirin, statin, or beta blockers.

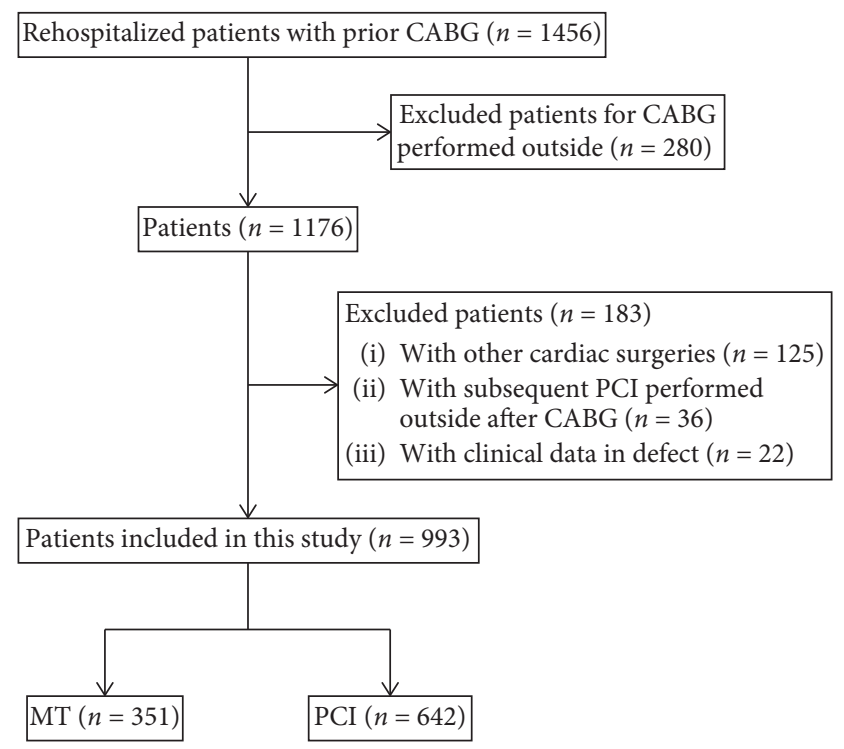

FIGURE 1: Outline of patients included and classified in this study. $\mathrm{CABG}=$ coronary artery bypass graft; $\mathrm{CAG}=$ coronary angiography; $\quad \mathrm{MT}=$ medical therapy; $\mathrm{PCI}=$ percutaneous coronary intervention.

TABle 1: Distribution of patients with recurrent angina accepting different treatments over duration after CABG $(n=993)$.

\begin{tabular}{|c|c|c|c|}
\hline Variables & MT & PCI & Total \\
\hline & $n=351$ & $n=642$ & $n=993$ \\
\hline $0-1 \mathrm{yr}$ & $40(11.4 \%)$ & $94(14.6 \%)$ & $134(13.5 \%)$ \\
\hline $1-5 \mathrm{yr}$ & $156(44.4 \%)$ & $298(46.4 \%)$ & $454(45.7 \%)$ \\
\hline $5-10 \mathrm{yr}$ & $117(33.3 \%)$ & $203(31.6 \%)$ & $320(32.2 \%)$ \\
\hline$>10 \mathrm{yr}$ & $38(10.8 \%)$ & $47(7.3 \%)$ & $85(8.6 \%)$ \\
\hline
\end{tabular}

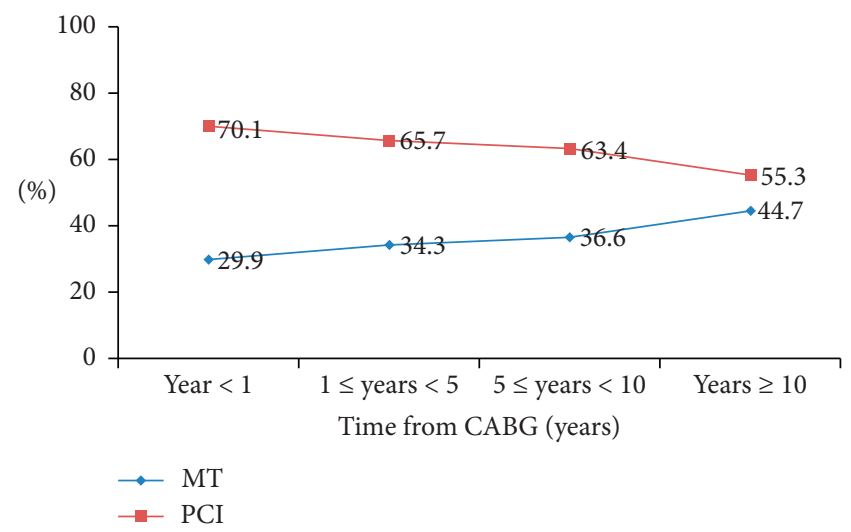

FIGURE 2: Distribution of patients accepting MT or PCI over different durations after CABG. MT vs PCI $P=0.014$. $\mathrm{CABG}=$ coronary artery bypass graft; $\mathrm{MT}=$ medical therapy; $\mathrm{PCI}=$ percutaneous coronary intervention.

3.3. CAG Characteristics of Diseased Grafts. The CAG characteristics of diseased grafts are shown in Table 3. There were 480 diseased grafts (stenosis and occlusion) in the MT group, accounting for $46.8 \%$ of 1025 grafts, which were lower than those 
TABLE 2: Comparison of baseline patient characteristics of symptomatic patients with prior CABG $(n=993)$.

\begin{tabular}{|c|c|c|c|}
\hline Variables & $\begin{array}{c}\text { MT } \\
n=351\end{array}$ & $\begin{array}{c}\text { PCI } \\
n=642\end{array}$ & $P$ value \\
\hline \multicolumn{4}{|l|}{ Demographics } \\
\hline Age (year) & $63.65 \pm 8.39$ & $62.20 \pm 8.81$ & 0.012 \\
\hline$\geq 65$ years & $166(47.3 \%)$ & $262(40.8 \%)$ & 0.052 \\
\hline Sex (male) & $257(73.2 \%)$ & $491(76.5 \%)$ & 0.281 \\
\hline \multicolumn{4}{|l|}{ Comorbidities } \\
\hline Diabetes & $168(47.9 \%)$ & $312(48.6 \%)$ & 0.842 \\
\hline Hypertension & $269(76.6 \%)$ & $482(75.1 \%)$ & 0.643 \\
\hline Dyslipidemia & $171(48.7 \%)$ & $313(48.8 \%)$ & 1.000 \\
\hline Chronic renal disease & $15(4.3 \%)$ & $24(3.7 \%)$ & 0.733 \\
\hline Chronic lung disease & $23(6$. & $22(3$. & 0.026 \\
\hline Prior PVD & $29(8.3$ & $68(10$ & 0.264 \\
\hline Prior CVA & $44(12$. & $108(16$. & 0.080 \\
\hline Prior MI & $31(8.8 \%)$ & $109(17.0 \%)$ & $<0.001$ \\
\hline Prior HF & $8(2.3$ & & 0.291 \\
\hline & & $33(5.1 \%)$ & 0.643 \\
\hline Smoking & $208(59.3 \%)$ & $412(64.2 \%)$ & 0.132 \\
\hline BMI & $26.30 \pm 3.21$ & $25.91 \pm 3.06$ & 0.132 \\
\hline Symptoms & & & 0.415 \\
\hline Chest pain & $333(94.9 \%)$ & $617(96.1 \%)$ & 0.035 \\
\hline SA & $142(42.6 \%)$ & $236(38.2 \%)$ & \\
\hline UA & $137(41.1 \%)$ & $237(38.4 \%)$ & \\
\hline AMI & $54(16.2 \%)$ & $144(23.3 \%)$ & \\
\hline Other & $18(5.1 \%$ & $25(3.9 \%)$ & \\
\hline Mean LVEF\% & $58.88 \pm 10.03$ & $58.69 \pm 9.35$ & 0.790 \\
\hline Mean LVEF\%※ & $55.24 \pm 9.26$ & $56.76 \pm 10.94$ & 0.411 \\
\hline Mean LVEF\% ${ }^{\S}$ & $59.68 \pm 10.32$ & $59.98 \pm 7.75$ & 0.780 \\
\hline Duration after CABG & $5.11 \pm 3.58$ & $4.59 \pm 3.42$ & 0.024 \\
\hline \multicolumn{4}{|l|}{ Medication after CABG } \\
\hline Aspirin & $289(82.3 \%)$ & $504(78.5 \%)$ & 0.160 \\
\hline Statin & $130(37.0 \%)$ & $224(34.9 \%)$ & 0.533 \\
\hline Beta blockers & $244(69.5 \%)$ & $428(66.7 \%)$ & 0.394 \\
\hline
\end{tabular}

$\mathrm{AF}=$ atrial fibrillation; $\mathrm{AMI}=$ acute myocardial infarction; $\mathrm{BMI}=$ body mass index; $\mathrm{CABG}=$ coronary artery bypass graft; $\mathrm{CVA}=$ cerebrovascular accident; $\mathrm{HF}=$ heart failure; $\mathrm{LVEF}=$ left ventricular ejection fraction; $\mathrm{MT}=$ medical therapy; $\quad \mathrm{PCI}=$ percutaneous coronary intervention; $\mathrm{PVD}=$ peripheral $\quad$ vascular disease; $\quad \mathrm{VE}=$ ventricular extrasystole; $\mathrm{SA}=$ stable angina; $\mathrm{UA}=$ unstable angina; $\mathrm{UCG}=$ echocardiography; ${ }^{*}$ results obtained from patients with AMI; ${ }^{\S}$ results obtained from patients with SA.

in the PCI group (50.1\%). In each group, the proportion of diseased saphenous vein grafts (SVGs) was higher than that of arterial graft, and grafts which were anastomosed at LAD territory and RCA territory were more likely to be diseased. Patients in the MT group were more likely to have chronic total occlusion (CTO) in diseased grafts $(82.7 \%$ vs $74.8 \%, P=0.003)$ than those in the PCI group. Due to graft occlusion, there was a high proportion of diseased grafts in each group, in which the location of lesions could not be defined.

3.4. CAG Characteristics of Ischemic Territory and Relevant Native Coronary Arteries. Table 4 shows CAG characteristics of the ischemic territories. Patients in the MT group (53.0\%) were more likely to suffer one ischemic territory while patients in the PCI group were more likely to suffer two ischemic territories (45.2\%); patients in each group were less likely to suffer three ischemic territories ( $8.8 \%$ vs $14.3 \%)$,
TABLE 3: CAG characteristics of diseased grafts in symptomatic patients with prior CABG (1408 diseased grafts in total 2877 grafts).

\begin{tabular}{lccc}
\hline Variables & MT & PCI & P value \\
\hline Diseased grafts & $46.8 \%(480 /$ & $50.1 \%(928 /$ & 0.067 \\
Type of diseased graft & $1025)$ & $1852)$ & 0.137 \\
$\quad$ Arterial graft & $76(15.8 \%)$ & $175(18.9 \%)$ & \\
SVG & $404(84.2 \%)$ & $753(81.1 \%)$ & \\
Anastomosis territory & & & 0.928 \\
LAD territory & $167(34.8 \%)$ & $321(34.6 \%)$ & \\
LCX territory & $129(26.9 \%)$ & $258(27.8 \%)$ & \\
RCA territory & $184(38.3 \%)$ & $349(37.6 \%)$ & \\
Anastomosis type & & & 0.525 \\
Individual & $168(35.0 \%)$ & $342(36.9 \%)$ & \\
Sequential & $312(65.0 \%)$ & $586(63.1 \%)$ & \\
Lesion characteristics & & & 0.003 \\
CTO & $397(82.7 \%)$ & $694(74.8 \%)$ & \\
Stenosis & $83(17.3 \%)$ & $234(25.2 \%)$ & $<0.001$ \\
70\%-80\% & $48(57.8 \%)$ & $66(28.2 \%)$ & \\
80\%-90\% & $18(21.7 \%)$ & $46(19.7 \%)$ & \\
90\%-99\% & $17(20.5 \%)$ & $121(51.7 \%)$ & \\
Lesion location & & & 0.002 \\
Aortic anastomosis & $61(12.7 \%)$ & $131(14.1 \%)$ & \\
Body & $49(10.2 \%)$ & $150(16.2 \%)$ & \\
Distal anastomosis & $85(17.7 \%)$ & $158(17.0 \%)$ & \\
Unknown & $285(59.4 \%)$ & $489(52.7 \%)$ & \\
\hline
\end{tabular}

$\mathrm{CABG}=$ coronary artery bypass graft; $\mathrm{CAG}=$ coronary angiography; $\mathrm{CTO}=$ chronic total occlusion; $\mathrm{LAD}=$ left anterior descending artery; $\mathrm{LCX}=$ left anterior descending artery; $\mathrm{MT}=$ medical therapy; $\mathrm{PCI}=$ percutaneous coronary intervention; $\mathrm{RCA}=$ right coronary artery; $\mathrm{SVG}=$ saphenous vein graft.

with a significant difference between two groups $(P=0.001)$. In one ischemic territory subgroup, in each group, patients were more likely to suffer RCA ischemic territory $(36.5 \%$ vs $40.8 \%$ ); in two ischemic territories subgroups, patients were more likely to suffer LCX ischemic territory and RCA ischemic territory $(67.2 \%$ in the MT group vs $62.8 \%$ in the PCI group).

The CAG characteristics of NCA relevant to ischemic territory are also shown in Table 4 . In the MT group, $80.6 \%$ of patients had type C CAD (severe lesions, based on ACC/ AHA Classification of CAD) and no patient had type A CAD, which were obviously different from that in the PCI group $(P<0.001)$. Compared to the PCI group, patients in the MT group were more likely to suffer diffuse lesions (49.3\% vs $15.0 \%, P<0.001)$. There were no significant differences in CTO lesions, lesions involving coronary artery branches, or openings between two groups.

3.5. Supplemental Data of CAG Characteristics and Progression of NCAs. CAG characteristics and progression of native coronary arteries before CABG and at the time of rehospitalization are shown in Supplemental Table 1. Before CABG, $7.4 \%$ of patients had single vessel lesion, $34.5 \%$ had two vessel lesions, and $58.1 \%$ had three vessel lesions in the MT group; when symptoms recurred after CABG, CAG showed that $2.6 \%$ had single vessel lesion, $29.1 \%$ had two vessel lesions, and $68.4 \%$ had three vessel lesions, which 
TABLE 4: CAG characteristics of ischemic territory and relevant native coronary arteries in symptomatic patients with prior CABG $(n=993)$.

\begin{tabular}{lccc}
\hline Variables & MT & PCI & P value \\
& $n=351$ & $n=642$ & \\
\hline Ischemic territory & & & 0.001 \\
One territory & $186(53.0 \%)$ & $260(40.5 \%)$ & 0.658 \\
$\quad$ LAD & $66(28.0 \%)$ & $73(28.1 \%)$ & \\
LCX & $68(36.5 \%)$ & $81(31.2 \%)$ & \\
RCA & $134(38.2 \%)$ & $106(40.8 \%)$ & \\
Two territories & $22(16.4 \%)$ & $64(22.1 \%)$ & 0.378 \\
LAD + LCX & $22(16.4 \%)$ & $44(15.2 \%)$ & \\
LAD + RCA & $90(67.2 \%)$ & $182(62.8 \%)$ & \\
LCX + RCA & $31(8.8 \%)$ & $92(14.3 \%)$ & NS \\
Three territories & $31(100.0 \%)$ & $92(100.0 \%)$ & \\
LAD + LCX + RCA & & $<0.001$ \\
Classification of CAD & & & \\
Type A & $0(0.0 \%)$ & $244(38.0 \%)$ & \\
Type B & $68(19.4 \%)$ & $9(1.4 \%)$ & \\
Type C & $283(80.6 \%)$ & $389(60.6 \%)$ & \\
Lesion characteristics & & & \\
CTO & $192(54.7 \%)$ & $321(50.0 \%)$ & 0.163 \\
Diffuse lesions & $173(49.3 \%)$ & $96(15.0 \%)$ & $<0.001$ \\
Branches involved & $97(27.6 \%)$ & $201(31.3 \%)$ & 0.247 \\
Opening involved & $97(27.6 \%)$ & $195(30.4 \%)$ & 0.383 \\
\hline
\end{tabular}

$\mathrm{CABG}=$ coronary artery bypass graft; $\mathrm{CAD}=$ coronary artery disease; $\mathrm{CAG}=$ coronary angiography; $\mathrm{CTO}=$ chronic total occlusion; $\mathrm{LAD}=\mathrm{left}$ anterior descending artery; LCX $=$ left anterior descending artery; $\mathrm{MT}=$ medical therapy; $\mathrm{NS}=$ not significant $\mathrm{RCA}=$ right coronary artery; $\psi=$ ACC $/$ AHA Classification of CAD.

indicated the progression of disease in NCAs. This progression was also observed in the PCI group. There were no significant differences in the number of diseased NCAs between the two groups before CABG or at the time of rehospitalization.

Figure 3 shows the distribution of patients with newly developed lesion sites, and there was no significant difference between two groups. In each group, patients were more likely to have newly developed lesions both in NCA and graft, especially in the PCI group (58.1\%).

3.6. Supplemental Data of PCI Procedural Characteristics. In the PCI group, $86.0 \%$ of patients underwent PCI in only NCA and $8.4 \%$ underwent PCI in only graft. In patients with ACS, 59 patients (15.5\%) underwent PCI in graft, of which $22.0 \%$ had PCI in 17 occluded vein grafts (Supplemental Table 2).

\section{Discussion}

For all we know, this is the largest observational, singlecenter Chinese study to compare the CAG characteristics of MT versus PCI therapy for symptomatic patients with prior CABG. We find that symptomatic patients with prior CABG are more likely to have newly developed lesions both in the native coronary artery and graft, and patients receiving MT (35.3\%) likely had occluded grafts and type C coronary artery disease featuring as diffuse lesions.

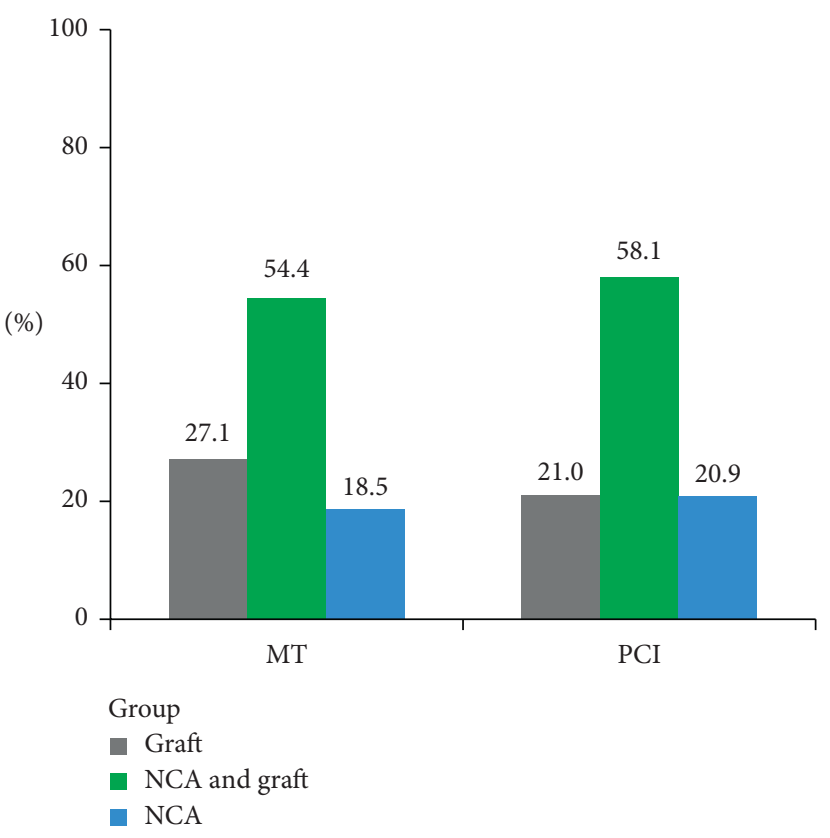

FIGURE 3: Distribution of patients with newly developed lesions in different vessels. MT vs PCI $P=0.073$. $\mathrm{MT}=$ medical therapy; $\mathrm{PCI}=$ percutaneous coronary intervention.

The occurrence of myocardial ischemia in patients with prior CABG might be caused by graft failure or disease progression of NCA [10]. Graft lesion rates of SVG and internal mammary artery (IMA) were about $20 \%-35 \%$ and $10 \%-20 \%$ within 5 years following CABG, respectively, and approximately $50 \%-75 \%$ and $20 \%-40 \%$ within 10 years following CABG $[2,11,12]$, respectively. Graft failure can be due to conduit defects, anastomotic technical errors, poor native vessel runoff or competitive flow with the native vessel, or atherosclerosis [13]. In this study, $78 \%$ of the patients were within 1 to 10 years following CABG, patients included in this study are those with recurrent symptoms after $\mathrm{CABG}$, and most patients have graft stenosis $>70 \%$ (81.5\% in the MT group, $79.1 \%$ in the PCI group); therefore, the proportion of patients with graft lesions is high. $44.1 \%$ of patients in the MT group and $38.3 \%$ of patients in the PCI group have graft age more than 5 years; with the prolongation of time after CABG, the risk of graft disease would also increase. When patients were discharged from hospital after $\mathrm{CABG}$, doctors usually recommended them to take aspirin, beta blockers, and statins throughout their life and control blood pressure and blood sugar. However, in the follow-up, we found that not all the patients in this study did persist in taking these drugs for some reasons like side effects or economic burden, and patients have always been poor in compliance with doctor's advice on medical therapy and lifestyle like exercise, smoking cessation, and low-fat diet. We also observe a high proportion of patients with risk factors associated with atherosclerosis [14], including gender (male), hypertension, diabetes, and hyperlipidemia $[15,16]$. Besides, we cannot rule out that the high incidence of graft lesions is associated with the anastomotic technique of surgeons; after all, there is still a gap of medical level and 
surgical techniques between China and European or American countries.

Redo CABG in patients with prior CABG is more challenging than the first CABG in many aspects $[17,18]$ including poor basic physical condition, severe pericardial adhesion, and unavailable conduits, which would increase the risk of perioperative complications. Redo CABG is a therapeutic option for some symptomatic patients with prior CBAG, especially in those situations where surgical risk is acceptable; adequate grafts are available, and multiple graft lesions and total occlusions of native coronary arteries and larger amount of ischemic myocardium are present. Compared to redo CABG, PCI had irreplaceable advantages in symptomatic patients with prior CABG, including lower risk of procedural mortality $[19,20]$, the similar long-term outcome [8], and low medical expense. However, PCI in patients with CTO should be considered against the risk of greater contrast volume, longer fluoroscopy time, and higher MACE rates in comparison with non-CTO patients.

When patients have recurrent symptoms after CABG surgery, they expect to get positive treatment than patients with initial angina, which may be related to their sensitivity to fear and pain of disease. In this study, some patients with stable angina also received PCI, and the choice of therapy depended mainly on doctors but also on the opinions of patients or their family members. Patients receiving MT are older and have more comorbidities; $80.6 \%$ had type $\mathrm{C}$ lesion featuring as diffuse lesions, which are often companied with extensive atherosclerotic and calcified vessels and longer lesions [21], leading to fewer amenable options for reintervention and suboptimal stent expansion [3]; we reviewed the data of patients with occluded vein grafts in the MT group; cardiologists tried CTO PCI in 22 patients, but all failed at last. Clinically, it was difficult to perform PCI in these patients for high procedural risks and technical-demanding complexity. We did not perform follow-up to these patients who accepted different treatments to further assess outcomes.

\section{Limitations}

Firstly, this study was a retrospective observational study; therefore, it is subject to all the limitations of observational studies. Secondly, the angiography film results were analyzed by one cardiac surgeon and one cardiologist. Thirdly, the classification of graft lesions was in reference to the evaluation criteria of native vessels. Fourthly, the decision to perform PCI for each patient was taken by two operators, based on an evaluation of CAG during CAG. Fifthly, we were just analyzing the clinical characteristics of patients receiving different treatments, especially coronary angiography. We had not yet studied the perioperative complications and follow-up outcomes of these patients treated with different treatments. Sixthly, due to the limitation of medical level and surgical techniques in Beijing Anzhen Hospital in China, we cannot rule out this condition that there might be some unreasonable aspects in surgical techniques and program of the first CABG which are associated with graft failure.

\section{Conclusions}

Symptomatic patients with prior CABG are more likely to have newly developed lesions both in the native coronary artery and graft. Most patients (64.7\%) could receive PCI. Patients receiving MT (35.3\%) likely had occluded grafts and type $\mathrm{C}$ coronary artery disease featuring as diffuse lesions.

\section{Data Availability}

The clinical data used to support the findings of this study are included within the supplementary information file.

\section{Conflicts of Interest}

The authors declare that they have no conflicts of interest.

\section{Authors' Contributions}

J. Wang obtained funding and designed the study. P. Chen, C. Zeng, M. Xin, Q. Ye, and Y. Zhao collected and analyzed the data. X. Ma drafted the manuscript. All authors have read and approved the final manuscript.

\section{Acknowledgments}

This study was supported by grants from the National Natural Science Foundation of China (81370294, 81570291, and 81770320).

\section{Supplementary Materials}

Supplemental Table 1: CAG characteristics and progression of native coronary arteries before CABG and at the time of rehospitalization. Supplemental Table 2: PCI procedural characteristics of symptomatic patients with prior CABG $(n=642)$. (Supplementary Materials)

\section{References}

[1] J. Escaned, "Secondary revascularization after CABG surgery," Nature Reviews Cardiology, vol. 9, no. 9, pp. 540-549, 2012.

[2] R. Scarsini, C. Zivelonghi, G. Pesarini, C. Vassanelli, and F. L. Ribichini, "Repeat revascularization: percutaneous coronary intervention after coronary artery bypass graft surgery," Cardiovascular Revascularization Medicine, vol. 17, no. 4, pp. 272-278, 2016.

[3] S. J. Brener, B. W. Lytle, I. P. Casserly, S. G. Ellis, E. J. Topol, and M. S. Lauer, "Predictors of revascularization method and long-term outcome of percutaneous coronary intervention or repeat coronary bypass surgery in patients with multivessel coronary disease and previous coronary bypass surgery," European Heart Journal, vol. 27, no. 4, pp. 413-418, 2006.

[4] D. L. Sprecher and G. L. Pearce, "How deadly is the "deadly quartet"?: a post-CABG evaluation," Journal of the American College of Cardiology, vol. 36, no. 4, pp. 1159-1165, 2000.

[5] C. A. Parasca, S. J. Head, M. Milojevic et al., "Incidence, characteristics, predictors, and outcomes of repeat revascularization after percutaneous coronary intervention and coronary artery bypass grafting: the SYNTAX trial at 5 years," 
JACC: Cardiovascular Interventions, vol. 9, no. 24, pp. 2493-2507, 2016.

[6] T. T. Michael, D. Karmpaliotis, E. S. Brilakis et al., "Impact of prior coronary artery bypass graft surgery on chronic total occlusion revascularisation: insights from a multicentre US registry," Heart, vol. 99, no. 20, pp. 1515-1518, 2013.

[7] L. Azzalini, S. Ojeda, A. Karatasakis et al., "Long-term outcomes of percutaneous coronary intervention for chronic total occlusion in patients who have undergone coronary artery bypass grafting vs those who have not," Canadian Journal of Cardiology, vol. 34, no. 3, pp. 310-318, 2018.

[8] D. A. Morrison, G. Sethi, J. Sacks et al., "Percutaneous coronary intervention versus repeat bypass surgery for patients with medically refractory myocardial ischemia: AWESOME randomized trial and registry experience with post-CABG patients," Journal of the American College of Cardiology, vol. 40, no. 11, pp. 1951-1954, 2002.

[9] Y. Nakamura, Y. Asaumi, T. Miyagi et al., "Comparison of long-term mortality in patients with previous coronary artery bypass grafting who underwent percutaneous coronary intervention with versus without optimal medical therapy," The American Journal of Cardiology, vol. 122, no. 2, pp. 206-212, 2018.

[10] T. Nakahara, M. R. Dweck, N. Narula, D. Pisapia, J. Narula, and H. W. Strauss, "Coronary artery calcification: from mechanism to molecular imaging," JACC: Cardiovascular Imaging, vol. 10, no. 5, pp. 582-593, 2017.

[11] L. Holmvang, B. Jurlander, C. Rasmussen, J. J. Thiis, P. Grande, and P. Clemmensen, "Use of biochemical markers of infarction for diagnosing perioperative myocardial infarction and early graft occlusion after coronary artery bypass surgery," Chest, vol. 121, no. 1, pp. 103-111, 2002.

[12] M. J. Price, N. Sawhney, J. A. Kao, A. Madrid, R. A. Schatz, and P. S. Teirstein, "Clinical outcomes after sirolimus-eluting stent implantation for de novo saphenous vein graft lesions," Catheterization and Cardiovascular Interventions, vol. 65, no. 2, pp. 208-211, 2005.

[13] F.-J. Neumann, M. Sousa-Uva, A. Anders et al., "2018 ESC/ EACTS guidelines on myocardial revascularization: the task force on myocardial revascularization of the European Society of Cardiology (ESC) and the European Association for Cardio-Thoracic Surgery (EACTS) developed with the special contribution of the European association of percutaneous cardiovascular interventions (EAPCI)," European Heart Journal, vol. 40, no. 2, pp. 1-96, 2019.

[14] M. Gaudino, C. Antoniades, U. Benedetto et al., "Mechanisms, consequences, and prevention of coronary graft failure," Circulation, vol. 136, no. 18, pp. 1749-1764, 2017.

[15] L. Panh, O. Lairez, J.-B. Ruidavets, M. Galinier, D. Carrié, and J. Ferrières, "Coronary artery calcification: from crystal to plaque rupture," Archives of Cardiovascular Diseases, vol. 110, no. 10 , pp. $550-561,2017$.

[16] A. J. Gassett, L. Sheppard, R. L. Mcclelland et al., "Risk factors for long-term coronary artery calcium progression in the multi-ethnic study of atherosclerosis," Journal of the American Heart Association, vol. 4, no. 8, Article ID e001726, 2015.

[17] H. Yaku and K. Doi, "Redo coronary artery bypass grafting," General Thoracic and Cardiovascular Surgery, vol. 62, no. 8, pp. 453-460, 2014.

[18] V. D. Bruno, M. Zakkar, F. Rapetto et al., "Early health outcome and 10-year survival in patients undergoing redo coronary surgery with or without cardiopulmonary bypass: a propensity score-matched analysis," European Journal of Cardio-Thoracic Surgery, vol. 52, no. 5, pp. 945-951, 2017.
[19] C.-H. Yap, L. Sposato, E. Akowuah et al., "Contemporary results show repeat coronary artery bypass grafting remains a risk factor for operative mortality," The Annals of Thoracic Surgery, vol. 87, no. 5, pp. 1386-1391, 2009.

[20] E. L. Fosbøl, Y. Zhao, D. M. Shahian, F. L Grover, F. H Edwards, and E. D Peterson, "Repeat coronary revascularization after coronary artery bypass surgery in older adults: the society of thoracic surgeons' national experience, 1991-2007," Circulation, vol. 127, no. 16, pp. 1656-1663, 2013.

[21] G. S. Guandalini and S. Bangalore, "The potential effects of new stent platforms for coronary revascularization in patients with diabetes," Canadian Journal of Cardiology, vol. 34, no. 5, pp. 653-664, 2018. 


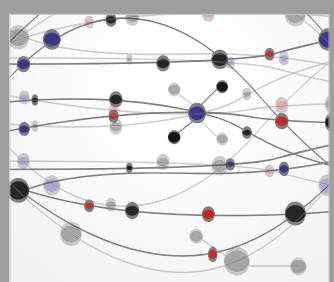

The Scientific World Journal
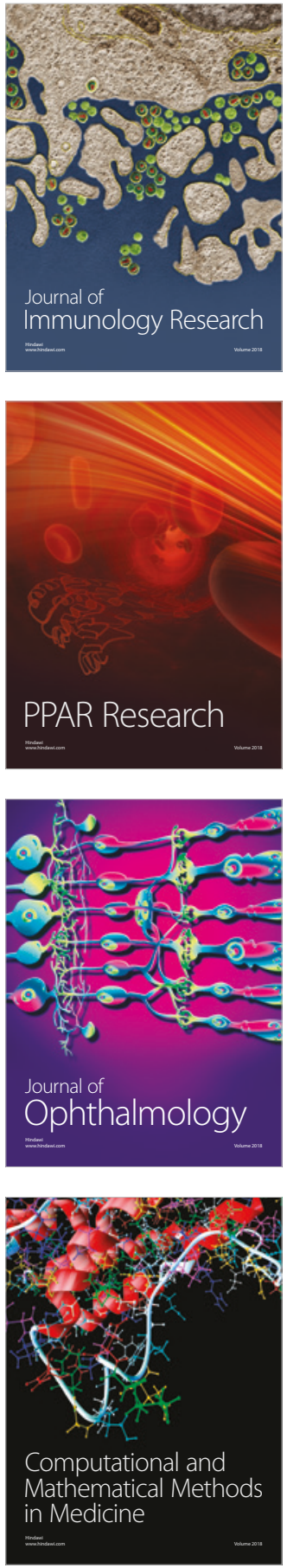

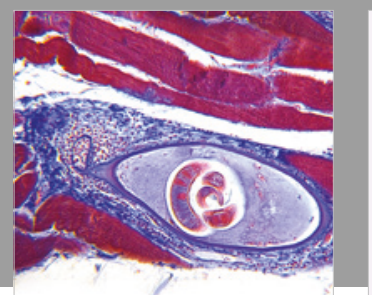

Gastroenterology Research and Practice

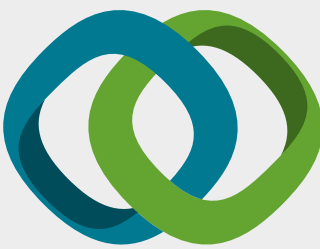

\section{Hindawi}

Submit your manuscripts at

www.hindawi.com
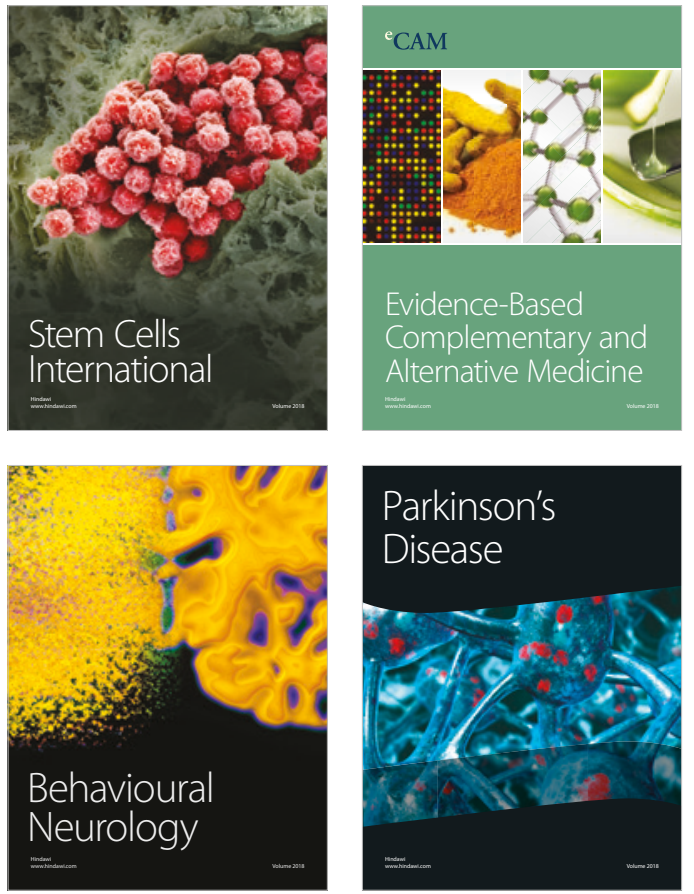

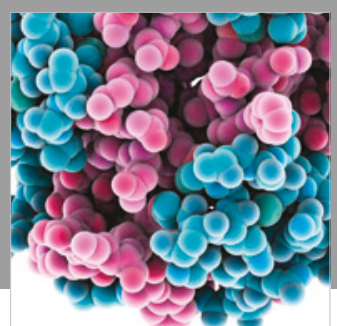

ournal of

Diabetes Research

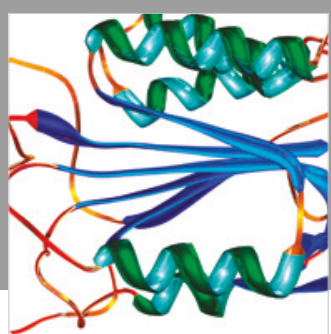

Disease Markers
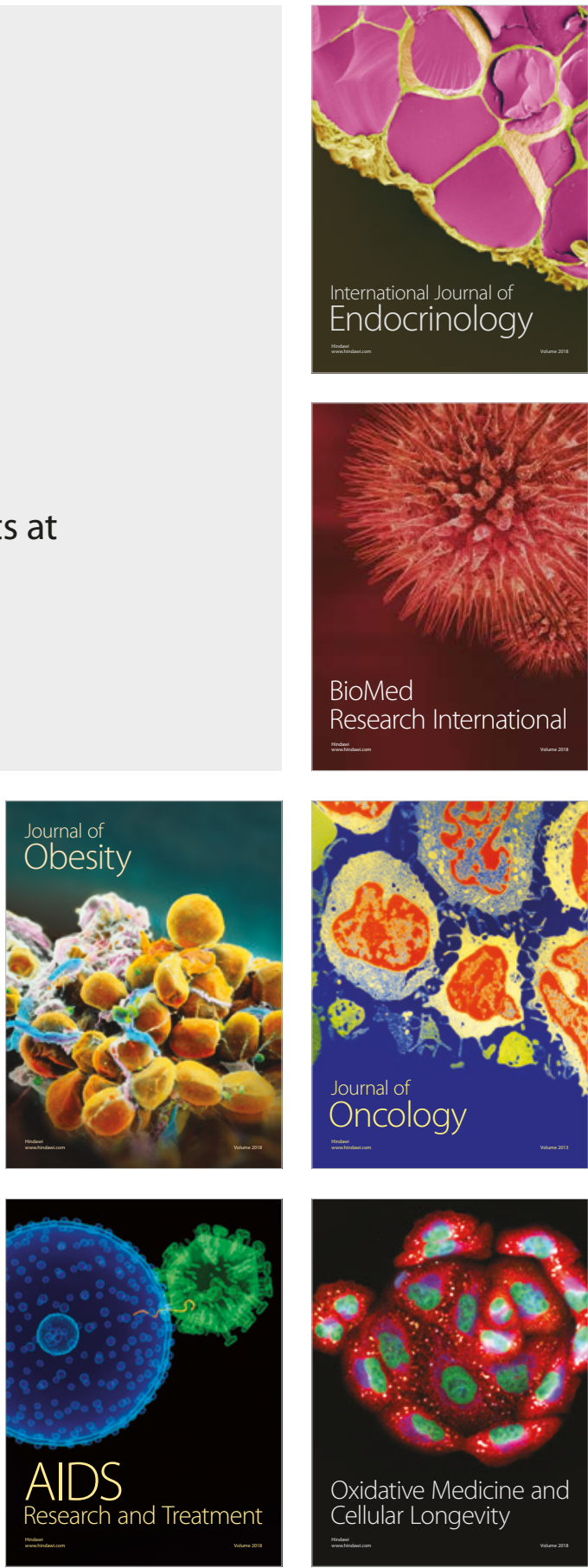